EUROPEAN ORGANIZATION FOR NUCLEAR RESEARCH

CERN - AB Department

CERN-AB-2007-072

CLIC Note 726

\title{
SUPERCONDUCTIVE DAMPING WIGGLERS FOR THE CLIC PROJECT
}

T. Baumbach ${ }^{2)}$, A. Bernard ${ }^{2)}$, H. Braun, S. Casalbuoni ${ }^{1)}$, A. Grau ${ }^{1)}$, M. Hagelstein ${ }^{1)}$, M. Korostelev, B. Kostka ${ }^{3)}$, E. Mashkina ${ }^{3)}$ A.-S. Mueller ${ }^{2}$, Y. Papaphilippou, R. Rossmanith ${ }^{1)}$, E. Steffens ${ }^{3)}$,

D. Wollmann ${ }^{2)}$, F. Zimmermann

CERN, Geneva, Switzerland

\begin{abstract}
The CLIC damping ring requires wigglers with both high on-axis fields and short periods. The present design foresees a superconductive wiggler with a period length of $5 \mathrm{~cm}$, a peak on-axis field of $2.5 \mathrm{~T}$ and a full aperture of $12 \mathrm{~mm}$. In this paper we explore the performance improvements of the damping ring when these parameters are pushed to $2.7 \mathrm{~T}$ at a period length of $2 \mathrm{~cm}$ with the expense of a reduced aperture of $5 \mathrm{~mm}$. A design for a prototype for testing the field quality of such a wiggler is presented in this paper and the possibility to test this wiggler with beam in the storage ring ANKA is described.
\end{abstract}

\footnotetext{
${ }^{1)}$ Institute for Synchrotron Radiation, Forschungszentrum Karlsruhe, Germany

${ }^{2)}$ University of Karlsruhe, Germany

${ }^{3)}$ University of Erlangen-Nürnberg, Germany
} 


\title{
SUPERCONDUCTIVE DAMPING WIGGLERS FOR THE CLIC PROJECT
}

\author{
S. Casalbuoni, A. Grau, M. Hagelstein, R. Rossmanith, Institute for Synchrotron Radiation, \\ Forschungszentrum Karlsruhe, Germany \\ A. Bernhard, A.-S. Mueller, D. Wollmann, T. Baumbach, University of Karlsruhe, Germany \\ B. Kostka, E. Mashkina, E. Steffens, University of Erlangen-Nürnberg, Germany \\ H. Braun, M. Korostelev, Y. Papaphilippou, F. Zimmermann, CERN, Switzerland
}

\begin{abstract}
The CLIC damping ring requires wigglers with both high on-axis fields and short periods. The present design foresees a superconductive wiggler with a period length of $5 \mathrm{~cm}$, a peak on-axis field of $2.5 \mathrm{~T}$ and a full aperture of $12 \mathrm{~mm}$. In this paper we explore the performance improvements of the damping ring when these parameters are pushed to $2.7 \mathrm{~T}$ at a period length of $2 \mathrm{~cm}$ with the expense of a reduced aperture of $5 \mathrm{~mm}$. A design for a prototype for testing the field quality of such a wiggler is presented in this paper and the possibility to test this wiggler with beam in the storage ring ANKA is described.
\end{abstract}

\section{CLIC DAMPING RING PARAMETERS}

Damping rings for linear collider have to maintain the vertical polarization of the injected beam. This condition is optimally fulfilled when the spin tune is exactly in between two integer depolarizing resonances:

$$
\left(\frac{g-2}{2}\right) \gamma=n+\frac{1}{2}
$$

$\gamma$ is the relative electron energy, $(\mathrm{g}-2) / 2$ is the gyromagnetic anomaly and $\mathrm{n}$ any integer. Equation (1) is fulfilled when the beam energy is $(n+1 / 2)$ times 440.7 $\mathrm{MeV}$ ( $\mathrm{n}$ any integer). For the CLIC damping ring an energy of $2.424 \mathrm{GeV}$ was chosen (spin tune of 5.5) [1].

Before entering the CLIC damping ring the beam is accelerated in a linac operating at $1.875 \mathrm{GHz}$ [2]. The RF frequency in the CLIC damping ring also will be 1.875 $\mathrm{GHz}$. The distance between buckets is $16 \mathrm{~cm}$. The proposed circumference of the ring is 300.48 or $364.96 \mathrm{~m}$ in the present design (1878 or 2281 buckets) and the machine will be filled with two trains of 110 bunches. Within one train each bucket is filled. Each bunch will consist of up to $2.6 \cdot 10^{9}$ electrons. The maximum stored current is in the order of $100 \mathrm{~mA}$. The injection and ejection procedure is repeated with a frequency of 150 Hz. Presently new parameters for CLIC are considered but not concluded. These changes will, however, not change the rational for the wiggler solution proposed here.

*Work supported partly by the German BMBF Research grant

ESAN05005407 and the $6^{\text {th }}$ EU Framework Program

${ }^{\#}$ Corresponding author, rossmanith@anka.fzk.de
The different circumferences $(300.48$ or $364.96 \mathrm{~m})$ are mainly due to different damping wiggler concepts [3]. The damping wigglers reduce the emittance. Since the bunch currents are relatively high intrabeam scattering (IBS) tries to increase the emittance. The effect for instance of the horizontal emittance growth due to IBS is described by the Piwinski formulation [4]:

$$
\frac{d \varepsilon_{x}}{d t}=-\frac{2\left(\varepsilon_{x}-\varepsilon_{x 0}\right)}{\tau_{x}}+\frac{2 \varepsilon_{x}}{T_{x}\left(\varepsilon_{x}, \varepsilon_{y}, \varepsilon_{z}\right)}
$$

$\varepsilon_{X}$ is the horizontal emittance in the presence of IBS, $\varepsilon_{x 0}$ is the unperturbed emittance defined by radiation excitation and radiation damping and low bunch population, $\tau_{X}$ is the horizontal radiation damping time and $T_{X}$ is the horizontal growth time due to IBS, which depends on the three beam emittances. Similar equations can be obtained for the vertical and longitudinal emittances, yielding a system of three coupled nonlinear differential equations, which can be solved numerically starting from the injected beam emittances.

A total circumference of $364.96 \mathrm{~m}$ is needed when the wigglers are made from permanent magnets (period length $10 \mathrm{~cm}$ and maximum field of $1.7 \mathrm{~T}$ ). The shorter circumference of $300.48 \mathrm{~m}$ is for superconducting wigglers with a period length of $4.5 \mathrm{~cm}$ and a maximum field of $2.52 \mathrm{~T}$.

Higher wiggler fields and shorter period lengths reduce the total length of the wiggler section and therefore the costs of the damping rings. They may also result in smaller beam emittances. In this paper we explore the performance improvements of the damping ring when these parameters are pushed to $2.7 \mathrm{~T}$ at a period length of $2 \mathrm{~cm}$ with the expense of a reduced aperture of $5 \mathrm{~mm}$.

Such a wiggler prototyp could be installed and tested in the storage ring ANKA. The nominal operating energy of ANKA is $2.5 \mathrm{GeV}$ [5] (injection energy $0.5 \mathrm{GeV}$ ) and very close to the required $2.424 \mathrm{GeV}$ and the presently achievable maximum current is $200 \mathrm{~mA}$. The existing RF frequency at ANKA is $500 \mathrm{MHz}$ and the circumference of the accelerator is $110.4 \mathrm{~m}$. The installation of a third harmonic system at ANKA is planned. In addition a triode type electron gun will be installed so that ANKA can be filled with any requested filling pattern (for instance single bunches). 


\section{THE INFLUENCE OF THE DAMPING WIGGLER PARAMETERS ON THE EMITTANCE}

Fig. 1 shows the calculated normalized emittance $\gamma \cdot \varepsilon_{X}$ in $\mathrm{nm}$ for a fixed wiggler length of $152 \mathrm{~m}$ for various wiggler parameters [2]. From this picture it is obvious that the best wigglers are those with a short period length $\lambda_{w}$ and a high field strength. As already mentioned superconductive wigglers are preferred over permanent magnet wigglers . Short period also means that wigglers with a small gap are more suitable for damping purposes.

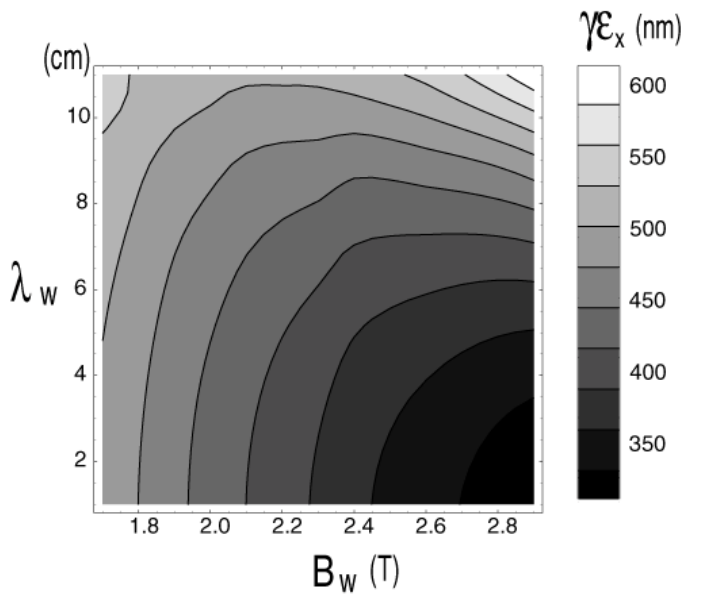

Fig. 1 Normalized emittance as a function of the wiggler parameters period length and field. The total length of the wiggler is $152 \mathrm{~m} \mathrm{[2].}$

With a gap of $5 \mathrm{~mm}$, a period length of $21 \mathrm{~mm}$ and a current density of $1 \mathrm{kA} / \mathrm{mm}^{2}$ a maximum magnetic field strength of $2.7 \mathrm{~T}$ can be achieved [6] leading to a normalized emittance of $350 \mathrm{~nm}$. Since two years a superconductive undulator with a gap of $8 \mathrm{~mm}$ is installed in ANKA [7]. A further reduction of the vertical betafunction will allow in future the operation with a gap of 5 $\mathrm{mm}$. The horizontal emittance in ANKA is at the moment $40 \mathrm{~nm}$ with a vertical emittance coupling of about $0.6 \%$. Since lifetime in a damping ring is not an issue operating of such a machine with a gap of $5 \mathrm{~mm}$ or even less should not be a problem, provided the aperture is large enough for the injected beam.

\section{THE WIGGLER CONCEPT}

A short version of the wiggler with a few periods is shown in fig. 2. The real wiggler will have a length of about 100 periods and is about $2.1 \mathrm{~m}$ long. The calculated field at the entrance of the damping element is shown in fig. 3. The corresponding $\mathrm{k}$-value is 5.26. Blue and green are bundles of superconductive wires. The different colors mark different current directions. A detailed description of the wiring technique can be found in [8]. Red is magnetic iron. ANKA presently uses a rectangular $\mathrm{NbTi}$ wire with a maximum current density between 1 to $1.1 \mathrm{kA} / \mathrm{mm}^{2}$. At the moment the University of Karlsruhe prepares a test with an undulator mock-up with $\mathrm{Nb}_{3} \mathrm{Sn}$ wire. The hope is that with this wire the maximum current can be significantly increased [9].

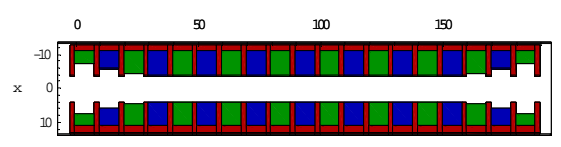

Fig. 2 Short version of the proposed wiggler with a gap of $5 \mathrm{~mm}$ and a period length of $2.1 \mathrm{~cm}$. Green and blue are superconductive wire bundles. The different colors mark different current directions. The corresponding field is shown in Fig. 3.

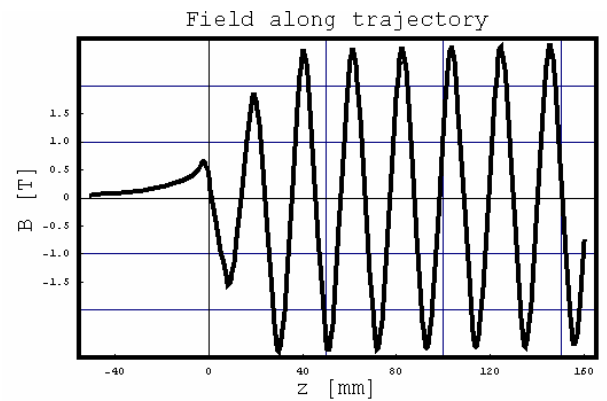

Fig. 3 Field of the superconducting wiggler shown in Fig. 2. The period length is $21 \mathrm{~mm}$, the gap is $5 \mathrm{~mm}$, the current $1 \mathrm{kA} / \mathrm{mm}^{2}$ and $\mathrm{K}=5.26$

\section{THE ARRANGEMENT OF THE WIGGLERS}

One of the most difficult problems with arrays of wigglers is the extraction and absorption of synchrotron radiation. This is especially a problem for superconductive wigglers. A possible solution is shown in Fig. 4.

Two undulators are in one cryostat (Fig. 4). The overall length of the cryostat will be about $5 \mathrm{~m}$. The vertical half opening angle of the radiation is $1 / \gamma$ and in 5 $\mathrm{m}$ distance the vertical size of the synchrotron beam is about $2 \mathrm{~mm}$. The horizontal opening angle is 5.26 times larger: $10.5 \mathrm{~mm}$. Integrated into the last part of the undulator is a $10 \mathrm{~cm}$ long vertical bending magnet with a relatively weak field of about $0.5 \mathrm{~T}$. This magnet deflects the electron beam by about $6 \mathrm{mrad}$. Photon beam and electron beam are separated by $3 \mathrm{~mm}$ after half a meter. The separation is sufficient to install an absorber after half a meter. After passing the section with the absorber the beam is deflected back to its previous vertical position. The parameters of the vertical bends must be chosen such that the vertical emittance generated by synchrotron radiation is small compared with the CLIC target value. 
As foreseen in any other solution the optics of the undulator section is a FODO channel. The quadrupoles can be either integrated into the cold section or can be normal room-temperature quadrupoles. The original version of the FODO channel requires a quadrupole after each wiggler and the maximum vertical beta-function is 7 $\mathrm{m}$. If this layout is adopted the quadrupole has to be integrated into the cold section.

One of the advantages of a superconductive wiggler is that without any mechanical motion the wiggler field can be reduced or completely switched off. This allows to bring the beam through the machine in a straightforward manner.

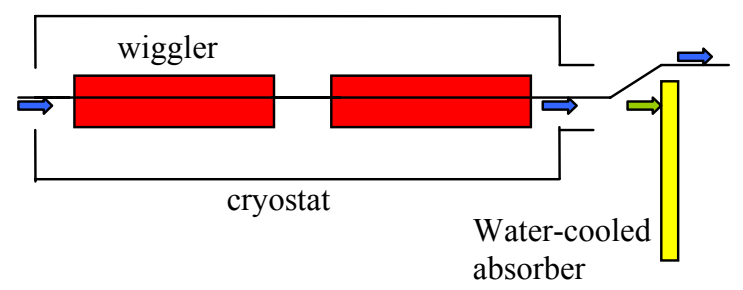

Fig. 4 Schematic layout of one wiggler unit for the CLIC damping rings. Two wigglers are in one cryostat. In between the cryostats is a warm section with an water cooled absorber. Electron beam and photon beam are separated by a soft vertical deflection.

\section{TEST OF THE WIGGLER PROTOTYPE AT ANKA}

Fig. 5 shows how the wiggler can be tested in ANKA. Without any changes it is possible to operate ANKA with a low vertical beta optics of $1.9 \mathrm{~m}$. The horizontal beta is $14 \mathrm{~m}$ and the horizontal emittance is $40 \mathrm{~nm}$. This allows to operate with a relatively long life time of 20 hours the ring with a gap of $8 \mathrm{~mm}$.

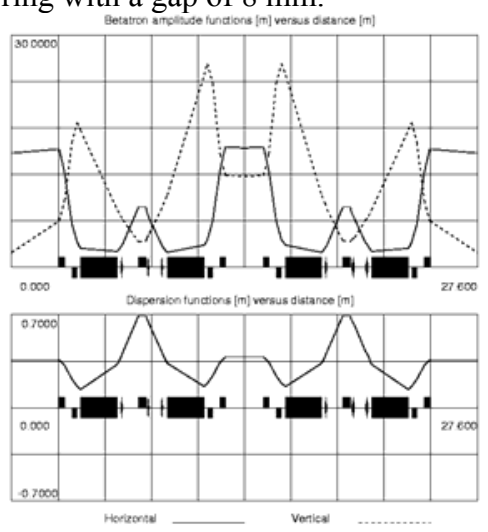

Fig. 5 Low vertical beta-optics in the long straight sections of ANKA: $\beta_{\mathrm{x}}=14 \mathrm{~m}, \beta_{\mathrm{y}}=1.9 \mathrm{~m}, \varepsilon_{\mathrm{x}}=40 \mathrm{~nm}$

During injection at $0.5 \mathrm{GeV}$ and subsequent energy ramping the gap has to be opened to about $25 \mathrm{~mm}$. This is a standard procedure for the ANKA insertion devices and the established mechanics can be adopted for this test undulator.

Fig. 6 shows that with these optics the lifetime will be significantly reduced. With a gap of $5 \mathrm{~mm}$ the beam life is expected to be 8 to 10 hours. Nevertheless, this lieftime is sufficient for operating ANKA in a damping ring mode and to study the influence of the damping wiggler on the beam.

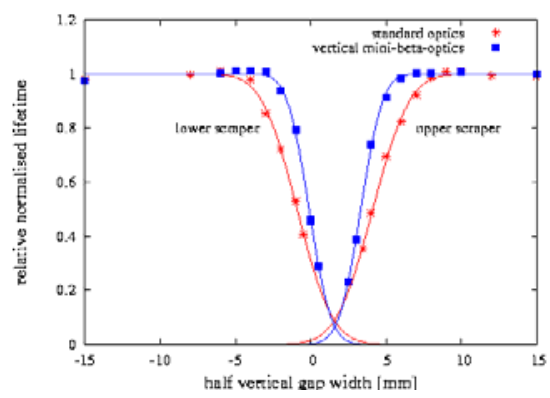

Fig. 6 Reduction of normalised lifetime for the optics shown in Fig. 5. The expected lifetime at ANKA is 8 to 10 hours with a gap of $5 \mathrm{~mm}$ [9].

\section{ACKNOWLEDGEMENTS}

This paper is based on discussions with many colleagues from CERN (especially the CLIC collaboration), from the Budker Institute, from the University and Research Center Karlsruhe, the University of Erlangen, Argonne National Lab. etc. The authors have to thank for all these contributions and discussions and apologize that due to the limited space it is impossible to list all the individual contributions.

\section{REFERENCES}

[1] M. Korostolev and F. Zimmermann, PAC 2002, Paris, http://accelconf.web.cern.ch/accelconf/

[2] M. Korostolev Ph. D. Thesis, CLIC-Note-701

[3] H. Braun, M. Korostelev, E. Levitchev, P. Piminov, D. Schulte, S. Siniatkin, P. Vobly, F. Zimmermann, K. Zolotarev, proc. EPAC, Edinburg, UK, 2006 and CLIC note 672

[4] A. Piwinski, Intra-Beam Scattering, Lecture Notes in Physics, 296, Joint US-CERN School (1986).

[5] ANKA Instrmentation Book, http://ankaweb.fzk.de/_file/extras/extras_download_3.pdf

[6] H. O. Moser, R. Rossmanith, NIMA Vol. 490, Sept. 2002, p. 403

[7] S. Casalbuoni et al., Phys. Rev. STAB, 9, 010702 (2006)

[8] R. Rossmanith, H. O. Moser, EPAC 2000, Vienna, Austria, http://accelconf.web.cern.ch/accelconf/I.

[9] B. Kostka, PhD Thesis, University of Erlangen, unpublished 